INSTITUT NATIONAL DE LA STATISTIQUE ET DES ETUDES ECONOMIQUES

Série des Documents de Travail du CREST

(Centre de Recherche en Economie et Statistique)

\title{
$n^{\circ} \mathbf{2 0 0 3 - 4 0}$
}

\section{Substituabilités entre demandes de facteurs induites par les demandes de biens}

\author{
E. MALINVAUD
}

Les documents de travail ne reflètent pas la position de l'INSEE et n'engagent que leurs auteurs.

Working papers do not reflect the position of INSEE but only the views of the authors.

\footnotetext{
${ }^{1}$ INSEE-CREST.
} 


\title{
Substituabilités entre \\ demandes de facteurs induites par les \\ demandes de biens
}

Edmond Malinvaud

Résumé. Les réponses à diverses questions de macroéconomie appliquée dépendent des valeurs attribuées aux élasticités de substitution entre les demandes de facteurs, ces demandes étant agrégées toutes origines sectorielles confondues. Les élasticités en cause traduisent non seulement des substituabilités dans les secteurs de production, mais aussi des substituabilités provenant du système des fonctions de demande de biens, lesquelles réagissent aux variations des prix relatifs de ces biens. Un modèle d'une assez grande généralité aboutit à une relation liant les variations des prix des facteurs aux variations des demandes agrégées de ces facteurs. La relation est appliquée à certaines spécifications particulières, notamment pour le cas de deux facteurs à une économie CES et à une économie dotée d'un système non-homothétique de fonctions de demandes pour les biens. Ainsi la relation présentée dans l'article permet d'étudier comment, au niveau agrégé, les substituabilités entre facteurs dépendent non seulement des formes des fonctions de production et de demande de biens, mais aussi de l'hétérogénéité entre les jeux de paramètres caractérisant ces fonctions.

\section{Substitutabilities between factor demands as depending on demands for goods}

\begin{abstract}
Answers to various questions of applied macroeconomics depend on the values given to elasticities of substitution between the demands for factors, these demands being aggregated across productive sectors. Such elasticities reflect not only substitutabilities within productive sectors, but also substitutabilities arising from the system of demand functions for goods, showing how the latter demands react to changes in the relative prices of goods. A fairly general model leads to a relation linking changes in factor relative prices to changes in the aggregate demands for these factors. The relation is applied to particular specifications, especially for the case of two factors to a CES economy and to an economy with a nonhomothetic system of demand functions for goods. Thus, the relation exhibited here lends itself to studies about how, at the aggregate level, factor substitutabilities depend not only on the forms of production and goods demand functions, but also on heterogeneity across the set of parameters characterizing these functions.
\end{abstract}

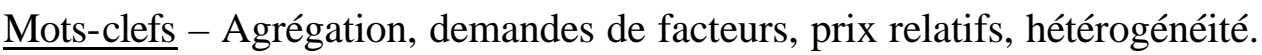

Classification JEL - E 24-25. 


\section{Substituabilités entre \\ demandes de facteurs induites par les \\ demandes de biens}

\section{Edmond Malinvaud}

Depuis longtemps un problème d'agrégation est en suspens dans la théorie de la production. Il concerne la relation entre, d'une part, les substitutions entre demandes de biens et, d'autre part, les substitutions entre demandes de facteurs productifs. Il implique l'hétérogénéité entre branches quant aux intensités des utilisations respectives des divers facteurs, de même que la complexité du système des courbes d'Engel qui régissent les demandes des biens produits par ces branches, cela pour tous ensembles de prix relatifs.

Déjà dans les années 1960 certains professeurs de macroéconomie enseignaient que les changements dans les prix des facteurs affectaient de deux façons la combinaison des facteurs au niveau agrégé : d'une part directement à cause de leurs effets sur la combinaison efficace à mettre en œuvre dans chaque entreprise, d'autre part indirectement, à travers les changements induits des prix relatifs des divers biens, à cause de leurs effets sur le panier des biens demandés. Souvent il était dit que les seconds effets s'ajoutaient aux premiers et que les substitutions entre facteurs étaient donc plus importantes au niveau agrégé que dans les unités de production. Motivé à l'origine par l'étude des allègements de charges sur les salaires des non-qualifiés, j'ai cherché à examiner de près la conjecture qui, de fait, se trouve être invalide dans toute une gamme de cas.

La pertinence d'une exploration analytique du sujet devrait être reconnue plus généralement qu'en relation avec l'étude des politiques visant à réduire le chômage des nonqualifiés. Par exemple elle s'applique à l'évaluation correcte des causes susceptibles d'expliquer les changements dans la prime à la qualification ${ }^{1}$. Mieux connaître comment calculer les effets indirects induits par les substitutions entre branches devrait être aussi pertinent pour savoir comment tirer parti en macroéconomie des ensembles de plus en plus riches d'estimations provenant de l'exploitation économétrique de bases de microdonnées d'entreprises.

Cette note utilise un modèle qui est délibérément réduit aux quelques éléments essentiels à faire intervenir dans une analyse locale du problème. Elle généralise grandement certains résultats obtenus dans E. Malinvaud (2001 et 2002). Pour la mettre au point j'ai beaucoup bénéficié d'échanges avec Alain Bernard qui s'est intéressé dans ses recherches aussi bien à la représentation des substituabilités qu'à l'agrégation ${ }^{23}$.

\footnotetext{
${ }^{1}$ Ainsi D. Acemoglu (2002) écrit : "the elasticity of substitution $\sigma$ [between skilled and unskilled labor] is important for the behavior of the skill premium when supply changes... Unfortunately this parameter is rather difficult to estimate, since it refers to an elasticity of substitution that combines substitution both within and across industries" (p. 20).

${ }^{2}$ Grâce à A. Bernard j'ai compris en particulier qu'il y avait une maladresse dans le traitement du modèle tel qu'il était présenté dans E. Malinvaud (2001 et 2002). Alors que j'avais procédé à une résolution complète des spécifications retenues, c'est-à-dire à une détermination de quasiment toutes les variations endogènes microéconomiques, avant d'agréger, il s'avère plus simple de procéder tôt à une agrégation partielle des relations induites par l'équilibre de la seule sphère productive (voir section 3 ici).
} 
L'étude procède à une analyse locale des variations de l'équilibre du modèle. Elle aboutit d'abord à une formule générale, dont sont ensuite tirées des implications, notamment grâce à l'examen de spécifications particulières. La formule, équation (39), caractérise les substituabilités entre demandes aggrégées de facteurs. Elle lie en effet les variations relatives des prix des facteurs à celle des consommations globales de ces facteurs (vecteurs $P$ et $X^{S}$ ). $\mathrm{Y}$ interviennent les caractéristiques de l'équilibre de référence quant à la structure de l'économie $\left(A^{h}\right.$ pour la technologie de la branche productive $h, C$ pour le système des courbes d'Engel) et quant à l'hétérogénéité microéconomique (entre les consommations unitaires $W^{h}$ de facteurs dans les branches et les élasticités-revenu $e_{h}$ des demandes de biens).

Le cas où n'existe que deux facteurs illustre bien les enseignements à tirer de cette étude, car les substituabilités s'y caractérisent par une seule grandeur : l'élasticité de substitution agrégée. Sa détermination implique plus qu'une agrégation appropriée des élasticités de substitution des diverses branches, car elle doit aussi prendre en compte les effets indirects dûs aux substitutions entre demandes de biens. Sans entrer pour le moment dans l'étude qui montre l'importance des moments centrés d'ordre 2 de la distribution statistique des $W^{h}$ et $e_{h}$, il s'avère que l'élasticité de substitution agrégée peut être sensiblement supérieure à une moyenne naturelle des élasticités de substitution dans les branches, mais cela si et seulement si d'une part l'hétérogénéité microéconomique est importante, d'autre part les substituabilités entre biens sont plus intenses que les substituabilités entre facteurs dans les branches.

Après qu'ait été posé le modèle (section 1), puis traité de l'analyse locale d'une branche productive (section 2), la section 3 aboutira à un système de relations entre les variations des demandes agrégées de facteurs et les variations des productions et des prix. La section 4 tirera parti des fonctions de demande des biens pour dégager une formule générale entre variations des demandes globales de facteurs et variations de leurs prix. L'interprétation de cette formule n'est pas évidente, mais elle sera éclairée d'abord par l'application au cas où n'existent que deux facteurs (section 5), puis par l'examen plus précis de deux spécifications particulières (sections 6 et 7).

\section{$\underline{\text { 1. Modèle et notation }}$}

Il existe $n$ biens ( $h=1,2 \ldots n)$ produits dans $n$ secteurs distincts, directement à partir de $m$ facteurs de production $(i=1,2 \ldots m)$ selon une technologie à rendements d'échelle constants ${ }^{4}$. La production du bien $h$ est dénotée $y_{h}$ et la quantité de facteur $i$ utilisée dans le secteur $h$ est dénotée $x_{i}^{h}$. Les marchés des biens et des facteurs sont traités comme concurrentiels (sauf pour quelques commentaires concernant le marché du travail non-qualifié). A l'équilibre le prix du facteur $i$ est $p_{i}$, celui du bien $h$ est $q_{h}$.

\footnotetext{
${ }^{3}$ Ma recherche sur le problème a aussi profité de discussions avec B. Crépon, E. Girardin, G. Laroque, C. Meghir J.-S Pischke, C. Teulings et des remarques de lecteurs anonymes.

${ }^{4}$ L'hypothèse simplifie l'analyse. Elle ne semble pas en restreindre foncièrement la portée, à en juger par E. Malinvaud (2002) où des cas de rendements d'échelle décroissants sont aussi considérés. Généraliser systématiquement l'analyse de façon à ce qu'elle couvre aussi de tels cas est concevable, mais conduirait à y apporter pas mal de complications, et cela dès cette section.
} 
Avec l'hypothèse de rendements constants, le revenu global $r$ est tel que :

$$
r=\sum_{h=1}^{n} q_{h} y_{h}=\sum_{i=1}^{m} p_{i}\left[\sum_{h=1}^{n} x_{i}^{h}\right]
$$

Le système des fonctions de production est dénoté par :

$$
y_{h}=f^{h}\left(x_{1}^{h}, x_{2}^{h} \ldots x_{m}^{h}\right) \quad h=1,2 \ldots n
$$

Les fonctions $f^{h}$ sont supposées deux fois différentiables.

Le système des fonctions de demande de biens est dénoté par :

$$
\ln y_{h}=g^{h}\left(\ln q_{1}, \ln q_{2} \ldots \ln q_{n} ; \ln r\right) \quad h=1,2 \ldots n
$$

Par hypothèse il satisfait identiquement l'équation de budget, première égalité de (1). Les fonctions $g^{h}$ sont supposées différentiables, leurs dérivées étant notées $g_{k}^{h}$ par rapport à $\ln q_{k}$ et $g_{r}^{h}$ par rapport à $\ln r$. Nous désignerons par la suite $C$ la matrice carrée ayant les éléments $C_{h k}=-g_{k}^{h}$ et par $e$ le vecteur colonne ayant les composantes $e_{h}=g_{r}^{h}$. Nous introduirons également dans un moment une règle de normalisation des prix, de façon à faire disparaître l'arbitraire que comporterait autrement notre spécification, du fait de l'homogénéité des demandes de biens par rapport aux grandeurs nominales.

Dans le secteur de production $h$ l'hypothèse de concurrence parfaite implique que $y_{h}$ et les $x_{i}^{h}$ maximisent :

$$
q_{h} y_{h}-\sum_{i=1}^{m} p_{i} x_{i}^{h}
$$

pour les valeurs supposées fixes de $q_{h}$ et des $p_{i}$, ainsi que sous la contrainte de la fonction de production (2). Il en résulte que ces $m+1$ variables sont solution de :

$$
\begin{aligned}
& q_{h} f_{i}^{h}\left(x_{1}^{h}, x_{2}^{h} \ldots x_{m}^{h}\right)=p_{i} \quad i=1,2 \ldots m \\
& q_{h} y_{h}=\sum_{i=1}^{m} p_{i} x_{i}^{h}
\end{aligned}
$$

où $f_{i}^{h}$ est la dérivée de $f^{h}$ par rapport à $x_{i}^{h}$ et l'équation (6) résulte de (2) et de ce que $f^{h}$ est une fonction homogène de degré 1 . La condition du second ordre pour que la solution en $x^{h}$ de (5) et (6) soit un maximum local de (4) est que la matrice des dérivées secondes de $f^{h}$ soit semi-définie négative en $x^{h}$.

Pour une définition complète du modèle il convient encore de se donner des hypothèses quant aux marchés des facteurs. Dans cette note nous supposerons le plus souvent 
ces marchés concurrentiels et admettrons que les offres agrégées des facteurs sont exogènes, soit $x_{i}^{S}$ pour tout $i$. Les équations du modèle seront alors complétées par :

$$
\sum_{h=1}^{n} x_{i}^{h}=x_{i}^{S} \quad i=1,2 \ldots m
$$

Peu de modifications auraient à intervenir si d'autres hypothèses étaient introduites sur les marchés des facteurs, par exemple que le prix $p_{m}$ du dernier facteur soit exogène et l'offre $x_{m}^{S}$ partiellement inemployée. Nous y reviendrons d'ailleurs.

Le modèle ainsi défini comporte $m(n+1)+2 n+1$ variables endogènes, les $x_{i}^{h}, p_{i}, y_{h}, q_{h}$ et $r$, correspondant aux équations (5), (7), (2), (3) et (1). Une analyse locale, s'appliquant au voisinage d'un équilibre supposé atteint, concerne les différentielles (infiniment petites) des variables endogènes ( $d x_{i}^{h}$, etc.) qui correspondent à des variations infinitésimales exogènes (le plus souvent les $d x_{i}^{S}$ ).

Nous devrons bien entendu opérer avec des représentations matricielles. En particulier les différentielles des variables endogènes (sauf $r$ ) seront représentées par les vecteurscolonnes $X^{h}, P, Y, Q$ dont les composantes seront :

$$
X_{i}^{h}=\frac{d x_{i}^{h}}{x_{i}^{h}} \quad P_{i}=\frac{d p_{i}}{p_{i}} \quad Y_{h}=\frac{d y_{h}}{y_{h}} \quad Q_{h}=\frac{d q_{h}}{q_{h}}
$$

La règle retenue ici, pour la normalisation du système des prix sera la contrainte linéaire suivante sur le vecteur $Q$ :

$$
\sum_{h=1}^{n} v_{h} \frac{d q_{h}}{q_{h}}=0
$$

où $v_{h}$ sera la part du bien $h$ dans le marché des biens, soit :

$$
v_{h}=\frac{q_{h} y_{h}}{r}
$$

\section{$\underline{\text { 2. Analyse locale de l'équilibre du secteur } h}$}

Pour la simplicité des formules omettons, sauf exception, l'indice $h$ dans cette section. L'équilibre d'un secteur, pour une demande $y_{h}$ qu'il a à satisfaire, est déterminé par la solution des équations (5) et (2) qui lui correspondent. Il nous faut maintenant étudier le système obtenu par différentiation.

Commençons par donner la forme logarithmique à (5) soit :

$$
\log q+\log f_{i}=\log p_{i} \quad i=1,2 \ldots m
$$

et par différentiation : 


$$
\frac{1}{f_{i}} \sum_{j=1}^{m} f_{i j} d x_{j}+\frac{d q}{q}=\frac{d p_{i}}{p_{i}} \quad i=1,2 \ldots m
$$

où $f_{i j}$ est la dérivée seconde de $f$ par rapport à $x_{i}$ et $x_{j}$.

De même (2) conduit à :

$\sum_{j=1}^{m} f_{j} d x_{j}=d y$

Pour la suite il convient d'introduire dès maintenant les variables auxiliaires :

$$
w_{i}^{h}=\frac{p_{i} x_{i}^{h}}{q_{h} y_{h}}=\frac{f_{i} x_{i}}{y}
$$

la seconde équation étant due à (5). Les $w_{i}$ sont les parts des différents facteurs dans le coût unitaire de la production du secteur, en conformité avec l'équation (6). Introduisons de plus la notation :

$$
b_{i j}=\frac{f f_{i j}}{f_{i} f_{j}}
$$

Avec ces notations les équations (12) et (13) deviennent :

$$
\sum_{j} b_{i j} w_{j} \frac{d x_{j}}{x_{j}}+\frac{d q}{q}=\frac{d p_{i}}{p_{i}} \quad \sum_{j} w_{j} \frac{d x_{j}}{x_{j}}=\frac{d y}{y}
$$

qui sont rassemblées dans la forme matricielle suivante :

$$
\left[\begin{array}{l}
P \\
d y / y
\end{array}\right]=\left[\begin{array}{ll}
B & U^{m} \\
{ }^{t} U^{m} & 0
\end{array}\right]\left[\begin{array}{l}
D_{w} X \\
d q / q
\end{array}\right]
$$

où $B$ est la matrice des $b_{i j}, U^{m}$ et ${ }^{t} U^{m}$ sont les vecteurs-colonnes et lignes ayant toutes leurs composantes égales à 1 , tandis que $D_{w}$ est la matrice diagonale des parts $w_{i}$.

Notons maintenant que pour tout $i$ :

$$
\sum_{j} f_{i j} x_{j}=0 \quad \quad \sum_{j} b_{i j} w_{j}=0
$$

La première équation, qui résulte de ce que $f_{i}$ est homogène de degré 0 , montre que 0 est racine de la matrice des dérivées secondes de $f$, associée au vecteur propre $x$. La seconde équation, qui résulte directement de la première et des définitions (14) et (15), montre de même que $w$ est vecteur propre de $B$ associé à la racine nulle. Notons encore que : 


$$
\sum_{i j} w_{i} b_{i j} w_{j}=\frac{1}{f} \sum_{i j} x_{i} f_{i j} x_{j} \leq 0
$$

où l'inégalité résulte de la condition du second ordre énoncée dans la section 1 . La matrice $B$ est donc semi-définie négative. Dès lors l'inverse de la matrice carrée figurant dans (17) existe $^{5}$ et a la forme :

$$
\left[\begin{array}{ll}
A & w \\
{ }^{t} w & 0
\end{array}\right]
$$

où $A$ est la matrice solution de :

$$
A B+w^{t} U^{m}=I \quad A U^{m}=0
$$

(Le fait que $B w$ soit le vecteur nul est donné par (18) tandis que ${ }^{t} w w=1$ résulte de (14) et de l'hypothèse de rendements constants).

Nous pouvons conclure cette section avec la relation matricielle déduite de (17), où nous réintroduisons l'indice $h$ :

$$
\left[\begin{array}{l}
D_{w}^{h} X^{h} \\
d q_{h} / q_{h}
\end{array}\right]=\left[\begin{array}{ll}
A^{h} & w^{h} \\
{ }^{t} w^{h} & 0
\end{array}\right]\left[\begin{array}{c}
P \\
d y_{h} / y_{h}
\end{array}\right]
$$

\section{Agrégation des demandes de facteurs}

Sur la forme (22) la nature d'un système analogue qui va lier les variations des demandes agrégées de facteur aux autres variations $d p_{i} / p_{i}, d y_{h} / y_{h}$ et $d q_{h} / q_{h}$ se devine assez aisément. En effet la iè̀me ligne s'écrit :

$$
w_{i}^{h} \frac{d x_{i}^{h}}{x_{i}^{h}}=\sum_{j=1}^{m} a_{i j}^{h} \frac{d p_{j}}{p_{j}}+w_{i}^{h} \frac{d y_{h}}{y_{h}}
$$

Or selon (7) :

$$
\frac{d x_{i}^{S}}{x_{i}^{S}}=\sum_{h=1}^{n} \frac{x_{i}^{h}}{x_{i}^{S}} \cdot \frac{d x_{i}^{h}}{x_{i}^{h}}
$$

où les rapports $x_{i}^{h} / x_{i}^{S}$ se calculent aisément à partir de (14) soit :

\footnotetext{
${ }^{5}$ Le lemme 1 de l'appendice de Diewert and Woodland (1977) l'indique, tout au moins si la matrice $B$ a le rang $m-1$, ce que nous pouvons supposer sans restreindre vraiment la portée de notre notre analyse. En effet la matrice $U^{m}{ }^{t} U^{m}-B$ est définie positive puisque, pour le seul vecteur propre $w$ de $B$ associé à la racine nulle, ${ }^{t} w U^{m}$ est égal à 1 .
} 


$$
p_{i} x_{i}^{h}=q_{h} y_{h} w_{i}^{h}
$$

donc

$$
p_{i} x_{i}^{S}=\sum_{h=1}^{n} q_{h} y_{h} w_{i}^{h}
$$

L'expression de droite a une interprétation directe si nous introduisons la part $v_{h}$ de chaque bien $h$ dans la valeur de la production globale, telle qu'elle a été définie par (10). Mise à part la valeur de cette production, le membre de droite est une moyenne pondérée de $w_{i}^{h}$. Nous pouvons écrire :

$$
\bar{w}_{i}=\sum_{h=1}^{n} v_{h} w_{i}^{h}
$$

et en déduire :

$$
\frac{x_{i}^{h}}{x_{i}^{S}}=\frac{v_{h} w_{i}^{h}}{\bar{w}_{i}}
$$

Ainsi, selon (24) :

$$
\bar{w}_{i} \frac{d x_{i}^{s}}{x_{i}^{S}}=\sum_{h=1}^{n} v_{h} w_{i}^{h} \frac{d x_{i}^{h}}{x_{i}^{h}}
$$

Cette dernière équation montre comment opérer pour obtenir un système qui va déterminer les variations relatives des demandes agrégées de facteurs, les $d x_{i}^{S} / x_{i}^{S}$ (la notation $x_{i}^{S}$ avait été introduite pour l'offre du facteur $i$, mais l'égalité entre offre et demande est supposée). S'agissant du facteur $i$ par exemple, il faut considérer les $n$ équations telles que (23) prises dans les divers secteurs, puis multiplier chacune par le poids $v_{h}$ lui correspondant, enfin sommer, pour avoir selon (29) la valeur de $\bar{w}_{i} d x_{i}^{S} / x_{i}^{S}$. Nous aboutissons ainsi à :

$$
\bar{w}_{i} \frac{d x_{i}^{S}}{x_{i}^{S}}=\sum_{j=1}^{m} a_{i j}^{S} \frac{d p_{j}}{p_{j}}+\sum_{h=1}^{n} v_{h} w_{i}^{h} \frac{d y_{h}}{y_{h}}
$$

où, bien entendu, les $a_{i j}^{S}$ sont les éléments de la matrice définie par :

$$
A^{S}=\sum_{h=i}^{n} v_{h} A^{h}
$$

Si $W$ désigne la matrice à $m$ lignes et $n$ colonnes des $w_{i}^{h}$, on aboutit ainsi au système : 


$$
\left[\begin{array}{r}
D_{\bar{w}} X^{S} \\
Q
\end{array}\right]=\left[\begin{array}{cc}
A^{S} & W D_{v} \\
{ }^{t} W & 0
\end{array}\right]\left[\begin{array}{l}
P \\
Y
\end{array}\right]
$$

où $D_{\bar{w}}$ et $D_{v}$ sont les matrices diagonales ayant respectivement les éléments $\bar{w}_{i}$ et $v_{h}$, tandis que $X^{S}$ a les éléments $d x_{i}^{S} / x_{i}^{S}$. (Les $n$ dernières lignes de (32) empilent les $(m+1)$-ème lignes des modèles sectoriels (22) dans l'ordre).

Des équations définies par $Q={ }^{t} W p$ qui apparaît sur (32) et de la règle de normalisation (9) on déduit directement

$$
0=\sum_{h} v_{h} \frac{d q_{h}}{q_{h}}=\sum_{h i} v_{h} w_{i}^{h} \frac{d p_{i}}{p_{i}}=\sum_{i} \bar{w}_{i} \frac{d p_{i}}{p_{i}}
$$

où il est tenu compte de (27). En d'autres termes la normalisation qui est retenue pour les variations des prix des biens impose une normalisation analogue sur les variations des prix des facteurs.

\section{$\underline{\text { 4. Demandes de biens et formule générale }}$}

Différentiant les fonctions de demande (3) nous obtenons :

$$
Y=-C Q+e \frac{d r}{r}
$$

Quelles conditions doivent être satisfaites par $C$ et $e$ pour que l'équation de budget (1) soit identiquement vérifiée par tout ensemble de variables obéissant à un tel système de lois de demande et à la règle de normalisation des prix ? Exprimée sur les différentielles l'équation (1) requiert :

$$
\frac{d r}{r}=\sum_{h} v_{h}\left[\frac{d y_{h}}{y_{h}}+\frac{d q_{h}}{q_{h}}\right]=\sum_{h} v_{h} \frac{d y_{h}}{y_{h}}
$$

où la seconde équation résulte de (9), c'est-à-dire de la normalisation des prix. Compte tenu de (34), (35) s'écrit :

$$
\frac{d r}{r}=\sum_{h} v_{h} e_{h} \cdot \frac{d r}{r}-\sum_{h k} v_{h} c_{h k} \frac{d q_{k}}{q_{k}}
$$

$C$ et $e$ seront tels que l'équation de budget soit toujours vérifiée si et seulement si :

$$
\sum_{h} v_{h} e_{h}=1 \quad \text { et } \quad \sum_{h k} v_{h} c_{h k} \frac{d q_{k}}{q_{k}}=0
$$

pour tout ensemble des $d q_{k} / q_{k}$ qui satisfera (9) ce qui impose l'existence d'un nombre $\gamma$ tel que : 


$$
\sum_{h} v_{h} c_{h k}=\gamma v_{k} \quad \text { pour tout } k
$$

Soit en termes matriciels :

$$
{ }^{t} v e=1 \quad{ }^{t} v\left[C-\gamma I^{h}\right]=0 \quad \text { pour un nombre } \gamma
$$

Ajoutons maintenant le système (34) aux relations de la section précédente. Les $n$ dernières lignes de (32) conduisent à :

$$
Y=e \frac{d r}{r}-C^{t} W P
$$

De plus la différentiation de (1) implique :

$$
\frac{d r}{r}=\sum_{i} \bar{w}_{i}\left[\frac{d x_{i}^{S}}{x_{i}^{S}}+\frac{d p_{i}}{p_{i}}\right]={ }^{t} \bar{w}\left[X^{S}+P\right]
$$

Ainsi :

$$
Y=e^{t} \bar{w} X^{s}+\left(e^{t} \bar{w}-C^{t} W\right) P
$$

Or les $m$ premières lignes de (33) s'écrivent :

$$
D_{\bar{w}} X^{S}=A^{S} P+W D_{v} Y
$$

Reportant dans cette équation la valeur de $Y$ donnée par (42) nous obtenons l'équation fondamentale de votre analyse.

$$
\left[D_{\bar{w}}-W D_{v} e^{t} \bar{w}\right] X^{S}=\left[A^{S}+W D_{v}\left(e^{t} \bar{w}-C{ }^{t} W\right)\right] P
$$

Elle rattache directement le vecteur $X^{S}$ des variations des demandes globales de facteurs au vecteur $P$ des variations des prix des facteurs. Tel était le but que nous visions. Il reste à bien interpréter le résultat obtenu.

Dès l'abord, quelques remarques générales peuvent être faites.

Remarque 1. Ce sont les seconds termes, à l'intérieur des deux crochets de la formule, qui capturent les effets indirects dûs aux variations induites sur les prix relatifs des biens et aux substitutions entre demandes de biens. S'il se trouvait que ces seconds termes soient nuls, alors la relation entre $X^{S}$ et $P$ s'exprimerait simplement par :

$$
D_{\bar{w}} X^{S}=A^{S} P
$$


qui transposerait directement celle apparaissant dans le secteur $h$ pour autant que n'interviendrait aucun effet volume (dans l'équation (22) la variation $d y_{h}$ serait alors nulle).

Remarque 2. On peut interpréter le second terme du membre de gauche de (44) comme traduisant un effet volume. En effet, s'il se trouvait que ${ }^{t} \bar{w} X^{S}$ soit nul, seul subsisterait dans le membre de gauche $D_{\bar{w}} X^{S}$. Or le cas :

$$
\sum_{i=1}^{m} \bar{w}_{i} \frac{d x_{i}^{S}}{x_{i}^{S}}=0
$$

correspond à un déplacement se faisant sur la surface isoquante dans l'espace des facteurs. C'est précisément ce que suppose la définition usuelle des élasticités de substitution entre facteurs, dans la théorie de la production ${ }^{6}$.

Remarque 3. S'il n'y avait pas d'hétérogénéité entre secteurs quant à leurs intensités d'utilisation respective des divers facteurs, c'est-à-dire si

$$
w_{i}^{h}=\bar{w}_{i} \quad i=1,2 \ldots m ; h=1,2 \ldots n
$$

alors la matrice $W$ serait égale à $\bar{w}^{t} U^{n}$. Le second terme du membre de droite de (44) n'aurait pas d'influence, ainsi que nous le verrons sur des spécifications plus précises. En somme, il n'y aurait pas d'effet induit sur les prix relatifs des biens, donc pas matière à substitution entre demandes de biens. Mais le cas qui nous intéresse est évidemment celui d'hétérogénéité.

Remarque 4. L'expression du second terme dans le crochet du membre de droite montre qu'il est une fonction quadratique de $W$. Cette remarque nous autorise à avancer, au moins provisoirement, la conclusion selon laquelle de petits écarts $w_{i}^{h}-\bar{w}_{i} \mathrm{n}$ 'auraient que des effets négligeables. Seuls risquent de vraiment importer les grands écarts.

Remarque 5. La forme du système (44) fait apparaître, comme multiplicateurs de $X^{S}$ et $P$ respectivement, deux matrices carrées d'ordre $m$. Elle suggère une résolution directe donnant en général la valeur du vecteur $P$ en fonction du vecteur exogène $X^{S}$. Mais l'équation (45) nous alerte, tout au moins pour le cas considéré dans la remarque 1, car la matrice symétrique $A^{S}$ est singulière, comme chacune des matrices $A^{h}$, du fait de leurs définitions dans lesquelles ${ }^{t} U^{m} A^{h}=0$ interviennent. Nous aurons effectivement à nous préoccuper de tels cas et à tenir compte alors de la normalisation des prix comme adjuvant nécessaire à une résolution complète du système. N'insistons pas plus sur ce point, au niveau de généralité où nous nous trouvons.

Pour préciser l'interprétation de la formule (44) il est éclairant de considérer des cas particuliers, ce que nous allons faire maintenant.

\footnotetext{
${ }^{6}$ Pour les discussions de la définition à donner aux élasticités de substitution voir Bernard (1986), Helm (1987) et les références qui y sont citées.
} 


\section{$\underline{\text { Facteurs multiples dans des économies presque log-linéaires }}$}

La formule (44) montre que les substituabilités entre demandes globales des divers facteurs dépendent d'une part des substituabilités dans les secteurs productifs et dans le système des demandes de biens (matrices $A^{h}$ et $C$ ), d'autre part de l'hétérogénéité entre secteurs dans l'utilisation des facteurs et entre demandes de biens (matrice $W$ et vecteur $e$ ). Dans les sections suivantes de cette note nous réduirons fortement le nombre de dimensions d'hétérogénéité en supposant $m=2$. Cela simplifiera beaucoup l'étude des conséquences des substituabilités microéconomiques.

Auparavant il convient de focaliser l'attention dans cette section sur un cas général quant au mombre $m$ des facteurs mais spécial par les fortes simplifications qu'il retient quant aux formes de la substituabilité dans les secteurs et dans le système des demandes de biens. Nous supposons pour cela que les fonctions de production $f^{h}$ sont log-linéaires (dites encore de Cobb-Douglas) et que le système des lois de demande fait intervenir une seule dimension de substituabilité. Pour celui-ci nous retenons le système CES («constant elasticity of substitution ») où n'intervient qu'une seule élasticité de substitution $\sigma^{C}$ commune à tous les choix bilatéraux entre biens et services. Cette spécification est employée très souvent en macroéconomie en raison de sa commodité analytique, sans doute d'ailleurs trop souvent. Dans le cas $\sigma^{C}=1$ le système des demandes de biens est lui-aussi log-linéaire.

Pour un secteur dont la fonction de production (2) est log-linéaire, nous écrivons :

$$
\log f=\sum_{i=1}^{m} \beta_{i} \log x_{i}
$$

où l'indice $h$ du secteur est pour l'instant omis et où les $\beta_{i}$ sont des constantes. Les élasticités de $f$ et de sa dérivée première $f_{i}$ par rapport à $x_{j}$ sont données par :

$$
\frac{x_{i} f_{i}}{f}=\beta_{i} \quad \frac{x_{i} f_{i j}}{f_{j}}=\beta_{i}-\delta_{i j}
$$

où $\delta_{i j}$ est l'indicateur de Kronecker. Ainsi $b_{i j}$ défini par (15) est égal à :

$$
b_{i j}=1-\frac{\delta_{i j}}{\beta_{i}}
$$

La matrice $A$ figurant dans (20), où les parts $w_{i}$ des facteurs sont égales aux paramètres $\beta_{i}$, est la solution de $(21)$, c'est-à-dire :

$$
\sum_{k} a_{i k} b_{k j}+\beta_{i}=\delta_{i j} \quad \sum_{k} a_{i k}=0
$$

Etant donné la forme (50) de $b_{i j}$, le calcul de $a_{i j}$ est immédiat : 


$$
a_{i j}=\beta_{i} \beta_{j}-\delta_{i j} \beta_{j}
$$

De même la matrice agrégée $A^{S}$ définie par (31) a les éléments donnés par :

$$
a_{i j}^{S}=\sum_{h} v_{h} \beta_{i}^{h} \beta_{j}^{h}-\delta_{i j} \sum_{h} v_{h} \beta_{j}^{h}
$$

Reprenant pour les moyennes les mêmes définitions que celles posées dans (27), nous ajoutons pour les covariances :

$$
\operatorname{Cov}\left(w_{i}, w_{j}\right)=\sum_{h} v_{h}\left(w_{i}^{h}-\bar{w}_{i}\right)\left(w_{j}^{h}-\bar{w}_{j}\right)
$$

Nous obtenons ainsi :

$$
a_{i j}^{S}=\operatorname{Cov}\left(\beta_{i}, \beta_{j}\right)+\bar{\beta}_{j}\left(\bar{\beta}_{i}-\delta_{i j}\right)
$$

Pour le système des lois de demande nous supposons :

$$
e=U^{n} \quad C=\sigma^{C} I^{n}
$$

$I^{n}$ étant la matrice unité d'ordre $n$. De plus nous écrivons $\sigma$ au lieu de $\sigma^{C}$ par simplicité. Pour l'application de la formule générale (44) nous pouvons partir de ce que :

$$
\left[W D_{v} e\right]_{i}=\bar{\beta}_{i} \quad\left[W D_{v} C{ }^{t} W\right]_{i j}=\sigma\left[\operatorname{Cov}\left(\beta_{i}, \beta_{j}\right)+\bar{\beta}_{i} \bar{\beta}_{j}\right]
$$

Le long d'une isoquante où ${ }^{t} \bar{\beta} X^{S}=0$, la formule (44) s'écrit donc, compte tenu de (56) :

$$
\overline{\beta_{i}} X_{i}^{S}=\sum_{j}\left\{(1-\sigma)\left[\operatorname{Cov}\left(\beta_{i}, \beta_{j}\right)+\overline{\beta_{i}} \bar{\beta}_{j}\right]-\overline{\beta_{j}} \delta_{i j}\right\}_{j}
$$

Avec la normalisation des prix donnée par (33) cette équation s'écrit plus simplement :

$$
\frac{d x_{i}^{S}}{x_{i}^{S}}=\frac{1-\sigma}{\bar{\beta}_{i}} \sum_{j} \operatorname{Cov}\left(\beta_{i}, \beta_{j}\right) \frac{d p_{j}}{p_{j}}-\frac{d p_{i}}{p_{i}}
$$

ou, de façon équivalente en notation matricielle :

$$
X^{S}=-\left[I^{n}-(1-\sigma) D_{\bar{w}}^{-1} C o v\right] P
$$

Ainsi la matrice des élasticités croisées, mises à part les élasticités directes liant par exemple $d x_{i}^{S} / x_{i}^{S}$ à $d p_{i} / p_{i}$, a la même structure que la matrice des covariances des $\beta_{i}$ entre secteurs. Dès lors que $m>2$ la description de la matrice des élasticités de substitution agrégées peut donner lieu à autant de développements que ceux dont a fait l'objet, en statistique mathématique, la description des matrices des covariances. 
De plus, nous notons immédiatement que le résultat est très simple dans l'économie intégralement log-linéaire $(\sigma=1)$ : si l'offre du facteur i augmente de 10 pour cent, le prix relatif de ce facteur diminue de 10 pour cent et les autres prix relatifs ne varient pas, cela quelles que soient sur l'isoquante les modifications compensatoires des autres facteurs. Cette économie est bien particulière. Le même résultat s'applique s'il n'y a pas d'hétérogénéité entre les secteurs, en ce sens que tous les moments du second ordre des $\beta_{i}^{h}$ sont nuls. Cas lui aussi bien particulier.

\section{$\underline{\text { 6. Le cas de deux facteurs seulement }}$}

Ce cas où $m=2$ a l'avantage d'apporter des résultats relativement simples dont l'interprétation est assez transparente. Avec $m>2$ se manifeste ce que les mathématiciens appellent parfois le «fléau de la dimensionnalité », qui dans un autre contexte a été bien révélé par le débat entre les deux Cambridge sur la théorie du capital. Ce fléau a pour effet de troubler les résultats et de nuire à la rigoureuse validité de toute interprétation simple.

La matrice $A^{S}$ représente les effets agrégés des substitutions s'effectuant à l'intérieur des secteurs de production. La déterminer suppose la solution, pour chaque secteur, du système (21). Complexe en général, ainsi que l'a montré A. Bernard (1986), cette solution est simple dans le cas $m=2$. Elle conduit alors à des résultats intuitivement satisfaisants et éclairants.

Comme nous avons supposé des rendements d'échelle constants dans la production, les fonctions de production (2) peuvent être exprimées sous la forme

$$
y_{h}=x_{1}^{h} \varphi^{h}\left[\frac{x_{2}^{h}}{x_{1}^{h}}\right] \quad h=1,2 \ldots n
$$

A partir de cette formule il est possible de déterminer l'expression suivante de la matrice $B^{h}$.

$$
B^{h}=\frac{-1}{\sigma^{h} w_{1}^{h} w_{2}^{h}}\left[\begin{array}{lr}
\left(w_{2}^{h}\right)^{2} & -w_{1}^{h} w_{2}^{h} \\
-w_{1}^{h} w_{2}^{h} & \left(w_{1}^{h}\right)^{2}
\end{array}\right]
$$

où $\sigma^{h}$ est la valeur de l'élasticité de substitution entre $x_{1}^{h}$ et $x_{2}^{h}$ au voisinage de l'équilibre considéré ${ }^{7}$.

La solution du système (21) conduit alors à une matrice $A^{h}$ ayant la forme simple suivante :

\footnotetext{
${ }^{7}$ Pour établir cette formule il convient évidemment de supprimer l'indice $h$ dans (43) et les dérivations à opérer. On démontre aisément que $w_{2}=x_{2} \varphi^{\prime} / x_{1} \varphi$ et $w_{1}=1-w_{2}$. La formule usuelle de l'élasticité de substitution d'une fonction de production à rendements constants permet d'écrire $\sigma=-x_{1}^{2} w_{1} w_{2} \varphi / x_{2}^{2} \varphi$ " où apparaît la dérivée seconde $\varphi^{\prime \prime}$. Après avoir calculé les dérivées $f_{1}, f_{2}, f_{11}, f_{12}, f_{22}$ de $f$, d'où les valeurs des $b_{11}, b_{12}$ et $b_{22}$, on aboutit à une matrice qui peut être exprimée sous la forme (44).
} 


$$
A^{h}=a^{h}\left[\begin{array}{ll}
-1 & 1 \\
1 & -1
\end{array}\right]
$$

où

$$
a^{h}=\sigma^{h} w_{1}^{h} w_{2}^{h}
$$

L'équation (31) implique alors que $A^{S}$ a une forme analogue à (65) avec :

$$
a^{S}=\sum_{h=1}^{n} v_{h} \sigma^{h} w_{1}^{h} w_{2}^{h}
$$

Le vecteur $A^{S} P$ a deux composantes égales en valeurs absolues mais de signes opposés. La première par exemple est égale à :

$$
a^{S}\left[\frac{d p_{2}}{p_{2}}-\frac{d p_{1}}{p_{1}}\right]
$$

Si nous admettons maintenant que les variations $d x_{1}^{S}$ et $d x_{2}^{S}$ se font le long d'une isoquante du plan $\left(x_{1}^{S}, x_{2}^{S}\right)$, nous voyons que le terme $A^{S} P$ dans le membre de droite de (44) conduirait, s'il était seul à intervenir à :

$$
\bar{w}_{1} \frac{d x_{1}^{S}}{x_{1}^{S}}=a^{S}\left[\frac{d p_{2}}{p_{2}}-\frac{d p_{1}}{p_{1}}\right]=-\bar{w}_{2} \frac{d x_{2}^{S}}{x_{2}^{S}}
$$

Ainsi nous aurions :

$$
\frac{d x_{1}^{S}}{x_{1}^{S}}-\frac{d x_{2}^{S}}{x_{2}^{S}}=\frac{a^{S}}{\bar{w}_{1} \bar{w}_{2}}\left[\frac{d p_{2}}{p_{2}}-\frac{d p_{1}}{p_{1}}\right]
$$

L'élasticité de substitution agrégée vaudrait alors :

$$
\hat{\sigma}^{S}=\sum_{h=1}^{n} v_{h} \frac{w_{1}^{h} w_{2}^{h}}{\bar{w}_{1} \bar{w}_{2}} \sigma^{h}
$$

donc une valeur qui n'est pas en général une moyenne pondérée ${ }^{8}$ des $\sigma^{h}$.

\footnotetext{
${ }^{8}$ En effet $\sum_{h} v_{h} w_{1}^{h} w_{2}^{h}=\bar{w}_{1} \bar{w}_{2}-\sum_{h} v_{h}\left(w_{1}^{h}-\bar{w}_{1}\right)^{2}$. Si les $\sigma^{h}$ étaient tous égaux à une même valeur $\sigma^{P}, \hat{\sigma}^{S}$ serait égal à $\sigma^{P}\left[1-\frac{\operatorname{Var}(w)}{\bar{w}_{1} \bar{w}_{2}}\right]$, où $\operatorname{Var}(w)$ est le second terme du membre de droite de l'équation précédente.
} 
Comme les seconds termes des crochets apparaissant dans l'équation (44) ne doivent pas en général être négligés, il faut que nous nous fassions une meilleure idée de leur rôle. Pour cela nous allons maintenir l'hypothèse $m=2$ et lui adjoindre d'autres hypothèses.

\section{Un système CES des demandes de biens}

Commençons par une spécification des demandes de biens. Nous retenons celle (57) déjà utilisée dans la section 5 et habituelle en théorie macroéconomique, où elle est souvent déduite d'une fonction d'utilité particulière attribuée au consommateur représentatif, la fonction CES à élasticité de substitution constante ${ }^{9}$.

Portons l'attention sur le second terme du crochet du membre droit de (44). L'élément $(i, j)$ de $W D_{v} C{ }^{t} W$ a l'expression :

$$
\sigma^{C} \sum_{h} v_{h} w_{i}^{h} w_{j}^{h}=\sigma^{C}\left[\operatorname{Cov}\left(w_{i}, w_{j}\right)+\bar{w}_{i} \bar{w}_{j}\right]
$$

avec la définition naturelle (54) de la covariance. De même l'élément $(i, j)$ de $W D_{v} e^{t} \bar{w}$ est égal à $\bar{w}_{i} \bar{w}_{j}$. Ainsi le système (44) s'écrit :

$$
\left[D_{\bar{w}}-\bar{w}^{t} \bar{w}\right] X^{S}=\left[A^{S}-\sigma^{C} \operatorname{Cov}\right] P-\left(\sigma^{C}-1\right) \bar{w}^{t} \bar{w} P
$$

où $C o v$ désigne la matrice $2 \times 2$ des covariances (54). Or la normalisation des variations sur le prix des facteurs (33) montre que le dernier terme de droite est nul. De plus, comme nous n'avons que deux facteurs et que $w_{1}^{h}+w_{2}^{h}=1$, nous voyons aisément que :

$$
\operatorname{Cov}\left(w_{1}, w_{2}\right)=-\operatorname{Var}\left(w_{1}\right)=-\operatorname{Var}\left(w_{2}\right)
$$

Ainsi

$$
-\operatorname{Cov}=\left[\begin{array}{cc}
-1 & 1 \\
1 & -1
\end{array}\right] \operatorname{Var}(w)
$$

En rapprochant cette formule de celle (64) s'appliquant à $A^{S}$, nous voyons que, au total, le système (72) prend la forme :

$$
\left[D_{\bar{w}}-\bar{w}^{t} \bar{w}\right] X^{S}=\left[a^{S}+\sigma^{C} \operatorname{Var}(w)\right]\left[\begin{array}{cc}
-1 & 1 \\
1 & -1
\end{array}\right] P
$$

A première vue on pourrait penser que cette expression simple confirme l'idée que l'élasticité de substitution agrégée tend à être systématiquement supérieure à une valeur

\footnotetext{
${ }^{9}$ Voir par exemple Malinvaud (2002), partie 4. On y note notamment que dans le système CES la règle de la normalisation (9) est équivalente à $d \hat{q} / \hat{q}=0$, où $\hat{q}$ est l'indice du niveau général des prix tel qu'il est habituellement défini dans ce système, car ses variations obéissent bien à : $\frac{d \hat{q}}{\hat{q}}=\sum_{h} v_{h} \frac{d q_{h}}{q_{h}}$.
} 
centrale des élasticités de substitution $\sigma^{h}$ des secteurs productifs. Mais cette impression est trompeuse, comme on le voit aisément si on considère le cas où tous les $\sigma^{h}$ seraient égaux à une même valeur $\sigma^{P}$ caractérisant la substituabilité entre les deux facteurs dans les productions. En effet dans ce cas, selon (66), $a^{S}$ serait égal à

$$
a^{S}=\sigma^{P} \sum_{h} v_{h} w_{1}^{h} w_{2}^{h}=\sigma^{P}\left[\bar{w}_{1} \bar{w}_{2}-\operatorname{Var}(w)\right]
$$

Ainsi une augmentation de l'hétérogénéité, exprimée ici par $\operatorname{Var}(w)$, ne se traduit par une augmentation du crochet figurant à gauche de (75) que si l'élasticité de substitution $\sigma^{C}$ dans la demande de biens est supérieure à l'élasticité de substitution $\sigma^{P}$ dans la demande de facteurs.

De fait, les égalités des deux vecteurs à deux composantes figurant à droite et à gauche de (75) s'écrivent grâce à une seule et même équation :

$$
\bar{w}_{1} \bar{w}_{2}\left[\frac{d x_{1}^{S}}{x_{1}^{S}}-\frac{d x_{2}^{S}}{x_{2}^{S}}\right]=\left[a^{S}+\sigma^{C} \operatorname{Var}(w)\right]\left[\frac{d p_{2}}{p_{2}}-\frac{d p_{1}}{p_{1}}\right]
$$

On lit directement sur cette équation que l'élasticité de substitution entre les deux facteurs au niveau agrégé est donnée par :

$$
\sigma^{s}=\frac{a^{s}+\sigma^{C} \operatorname{Var}(w)}{\bar{w}_{1} \bar{w}_{2}}
$$

Dans le cas particulier où les $\sigma^{h}$ sont tous égaux au même $\sigma^{P}$, la formule devient ${ }^{10}$ :

$$
\sigma^{S}=\sigma^{P}+\left(\sigma^{C}-\sigma^{P}\right) \frac{\operatorname{Var}(w)}{\bar{w}_{1} \bar{w}_{2}}
$$

L'équation (77) donne l'essentiel pour la solution complète du système. Ainsi, s'agissant de valeurs exogènes données des variations des offres de facteurs $d x_{1}^{S} / x_{1}^{S}$ et $d x_{2}^{S} / x_{2}^{S}$, les deux variations endogènes $d p_{1} / p_{1}$ et $d p_{2} / p_{2}$ sont déterminées individuellement par la normalisation des prix, l'équation (33), soit :

$$
\frac{d p_{1}}{p_{1}}=-\bar{w}_{2}\left[\frac{d p_{2}}{p_{2}}-\frac{d p_{1}}{p_{1}}\right] \quad \frac{d p_{2}}{p_{2}}=-\bar{w}_{1}\left[\frac{d p_{2}}{p_{2}}-\frac{d p_{1}}{p_{1}}\right]
$$

la valeur du crochet figurant à droite dans les deux équations étant déduite directement de (77).

De même, (77) et (80) donnent la solution pour le cas où, le second facteur étant par exemple le travail non-qualifié, il existerait une offre excédentaire de ce travail conjointement

\footnotetext{
${ }^{10}$ Cette dernière formule est conforme à celles établies dans E. Malinvaud (2002) à partir d'un modèle plus étroitement spécifié.
} 
avec un prix $p_{2}$ exogène. Dans (77) il faudrait alors remplacer $d x_{2}^{S} / x_{2}^{S}$ par la variation endogène de la demande globale $x_{2}^{D}$ de travail non-qualifié. En l'absence de toute variation de l'offre du premier facteur $x_{1}^{S}$, on obtient immédiatement :

$$
\frac{d x_{2}^{D}}{x_{2}^{D}}=\frac{-\left[a^{S}+\sigma^{C} \operatorname{Var}(w)\right]}{\bar{w}_{2}\left(\bar{w}_{1}\right)^{2}} \cdot \frac{d p_{2}}{p_{2}}
$$

L'effet de la substitution dans la demande de facteurs se trouve magnifié par le multiplicateur $1 / \bar{w}_{1}$, qui traduit la réduction du déséquilibre sur le marché des facteurs.

Au total la solution du système CES est très simple. Un peu de réflexion explique cette simplicité par le caractère intégralement homothétique du système. Non seulement intervient l'hypothèse de rendements d'échelle constants dans la production. Mais aussi celle, beaucoup plus irréaliste, d'homothétie du système des lois de demandes pour les biens : les courbes d'Engel sont toutes des droites issues de l'origine. La commodité analytique se paie donc d'un prix élevé.

Le réaliser nous incite à examiner un cas moins favorable, où la présence simultanée de biens de première nécessité et de biens de luxe serait reconnue. Cette préoccupation conduisit dès les années 1950s R. Stone (1954) et R. Geary à examiner, indépendamment l'un de l'autre, un système de lois de demande linéaires ayant cette caractéristique. Nous allons reprendre ce système sous une forme un peu plus générale où figurera une élasticité de substitution $\sigma^{C}$ quelconque (Stone et Geary avait considéré le cas $\sigma^{C}=1$ ).

\section{Un système non-homothétique des demandes de biens}

Contentons-nous ici de donner les grandes lignes de l'argumentation qui est plus précisément exposée en annexe. Le système des lois de demande est supposé avoir la forme :

$$
y_{h}=\chi_{h}+\gamma_{h}\left[\frac{q_{h}}{\hat{q}}\right]^{-\sigma^{c}} \frac{r_{e}}{\hat{q}}
$$

où $\chi_{h}, \gamma_{h}$ et $\sigma^{C}$ sont des paramètres, la somme des $\gamma_{h}$ étant égale à 1 . Le paramètre $\chi_{h}$ est positif pour un bien de première nécessité. La variable $\hat{q}$ est un indicateur du niveau général des prix défini par :

$$
\hat{q}^{1-\sigma^{c}}=\sum_{h=1}^{n} \gamma_{h} q_{h}^{1-\sigma^{c}}
$$

La variable $r_{e}$ est le «revenu excédentaire » :

$$
r_{e}=\sum_{h=1}^{n} q_{h}\left(y_{h}-\chi_{h}\right)
$$


Il est facile de voir que si tous les $\chi_{h}$ sont nuls, ce système est similaire à celui considéré dans la section précédente.

L'annexe montre que le nouveau système est un cas particulier de celui introduit dans la section 1 avec les spécifications suivantes pour les élasticités de revenu :

$$
e_{h}=\left[1-\frac{\chi_{h}}{y_{h}}\right] \frac{r}{r_{e}}
$$

les élasticités-prix - $C_{h k}$ étant alors définies par :

$$
C_{h k}=\frac{\sigma^{C} r_{e}}{r} e_{h} \delta_{h k}+e_{h} v_{k}\left[1-\frac{\sigma^{C} r_{e}}{r} e_{k}\right]
$$

où $\delta_{h k}$ est l'indice de Kronecker et $v_{h}$ est défini par (10). On note que $r_{e}=r$ et $e_{h}=1$ pour tout $h$ ramènent à la spécification (57).

Nous allons d'abord considérer le système des deux équations (44) pour des déplacements se faisant le long d'une isoquante ${ }^{t} \bar{w} X^{S}=0$. L'annexe montre que ce système se ramène à une équation (A.27) qui peut s'écrire :

$$
\bar{w}_{1} \bar{w}_{2}\left[\frac{d x_{1}^{S}}{x_{1}^{S}}-\frac{d x_{2}^{S}}{x_{2}^{S}}\right]=\left\{a^{S}+\frac{\sigma^{C} r_{e}}{r}\left[m\left(e w^{2}\right)-[m(e w)]^{2}\right]\right\}\left[\frac{d p_{2}}{p_{2}}-\frac{d p_{1}}{p_{1}}\right]
$$

où figurent les moments suivants de la distribution statistique, entre biens, des $e_{h}$ et $w_{1}^{h}$ :

$$
m(e w)=\sum_{h} v_{h} e_{h} w_{1}^{h} \quad m\left(e w^{2}\right)=\sum_{h} v_{h} e_{h}\left(w_{1}^{h}\right)^{2}
$$

Ainsi l'élasticité de substitution agrégée est donnée par :

$$
\sigma^{S}=\frac{a^{S}}{\bar{w}_{1} \bar{w}_{2}}+\sigma^{C} \frac{r}{r} \cdot \frac{m\left(e w^{2}\right)-[m(e w)]^{2}}{\bar{w}_{1} \bar{w}_{2}}
$$

Afin de rendre cette formule plus transparente, l'annexe introduit des moments centrés tels que :

$$
\bar{m}\left(e w_{1}\right)=\sum_{h} v_{h}\left(e_{h}-1\right)\left(w_{1}^{h}-\bar{w}_{1}\right)=\operatorname{Cov}\left(e, w_{1}\right)
$$

Elle concentre aussi l'attention sur le cas où le moment centré du troisième ordre $\bar{m}\left(e w_{1}^{2}\right)$, particulièrement difficile à évaluer et vraisemblablement très faible, serait nul. Elle dégage alors, pour l'élasticité de substitution agrégée $\sigma^{S}$, des formules qui peuvent se comparer à celles (78) et (79) s'appliquant à l'économie CES étudiée dans la section 7. Ainsi (78) se compare à : 


$$
\bar{w}_{1} \bar{w}_{2} \sigma^{S}=a^{S}+\frac{r_{e}}{r} \sigma^{C}\left[\operatorname{Var}(w)-[\operatorname{Cov}(e, w)]^{2}\right]
$$

Pour des valeurs données de $\bar{w}_{1}, \operatorname{Var}(w)$ et $a^{S}$, l'élasticité $\sigma^{S}$ aurait tendance à être plus faible dans l'économie de Stone-Geary que dans l'économie CES correspondante.

Mise à part l'interférence éventuelle de $\bar{m}\left(e w_{1}^{2}\right)$, le membre de droite de (91) est plus faible que celui de (78), à la fois parce que $\sigma^{C} \operatorname{Var}(w)$ est multiplié par $r_{e} / r$, normalement inférieure à 1 , et parce qu'intervient en déduction le terme normalement positif dû au carré de Cov $(e, w)$. Cependant ces deux corrections n'ont vraisemblablement qu'un impact assez faible. En particulier le rapport $[\operatorname{Cov}(e, w)]^{2} / \operatorname{Var}(w)$ est égal à la variance des élasticitésrevenu multipliée par le carré du coefficient de corrélation entre les $e_{h}$ et $w_{1}^{h}$.

En revanche si nous considérons, comme nous l'avons fait à la fin de la section précédente, l'effet des variations du prix $p_{2}$ du travail non-qualifié sur la demande globale $x_{2}^{D}$ de ce travail en cas d'offre excédentaire, la formule (81) doit être remplacée par une autre, (A.35) en annexe. Le multiplicateur, dû à la modification du déséquilibre sur le marché du travail non-qualifié, se révèle alors sensible à la corrélation intersectorielle entre le contenu en travail non-qualifié $\left(w_{2}^{h}\right)$ et l'élasticité-revenu de la demande de biens $\left(e^{h}\right)$. Plus ce contenu est relativement élevé pour les biens de luxe ( $e^{h}$ élevé), plus donc $\operatorname{Cov}(e, w)$ est négative, plus l'effet multiplicateur transitant par le marché des biens est lui aussi élevé ${ }^{11}$.

\footnotetext{
${ }^{11}$ Ce résultat bien naturel avait été annoncé dans Malinvaud (2001) pour le cas dans lequel la même élasticité de substitution $\sigma^{P}$ s'appliquait à tous les secteurs de production. La formule était toutefois moins transparente, car elle ne concernait pas directement les élasticités-revenu $e^{h}$.
} 


\section{Annexe}

\section{Traitement de la spécification de Stone-Geary}

Soit :

$$
y_{h}=\chi_{h}+\gamma_{h}\left[\frac{q_{h}}{\hat{q}}\right]^{-\sigma^{c}} \cdot \frac{r_{e}}{\hat{q}}
$$

les demandes de biens s'adressant aux divers secteur $h$, l'indicateur $\hat{q}$ étant défini par :

$$
\hat{q}^{1-\sigma^{c}}=\sum_{h} \gamma_{h} q_{h}^{1-\sigma^{c}}
$$

et le revenu excédentaire $r^{e}$ par :

$$
r_{e}=\sum_{h} q_{h}\left(y_{h}-\chi_{h}\right)
$$

(voir équations (82) à (84) du texte). Dans cette annexe nous écrirons maintenant $\sigma$ au lieu de $\sigma^{C}$ afin de simplifier les formules.

L'équation (A.1) peut se mettre sous la forme :

$$
\ln y_{h}+\ln \left[1-\frac{\chi_{h}}{y_{h}}\right]=-\sigma \ln q_{h}+\ln r_{e}+(\sigma-1) \ln \hat{q}+\ln \gamma_{h}
$$

Différentiant nous obtenons :

$$
\begin{aligned}
\frac{d y_{h}}{y_{h}}+\frac{\chi_{h}}{y_{h}}\left[1-\frac{\chi_{h}}{y_{h}}\right]^{-1} \frac{d y_{h}}{y_{y}} & =\left[1-\frac{\chi_{h}}{y_{h}}\right]^{-1} \frac{d y_{h}}{y_{h}} \\
& =-\sigma \frac{d q_{h}}{q_{h}}+\frac{d r_{e}}{r_{e}}+(\sigma-1) \frac{d \hat{q}}{\hat{q}}
\end{aligned}
$$

De même (A.3) conduit à :

$$
\frac{d r_{e}}{r}=\frac{d r}{r}-\sum_{k} \frac{\chi_{k}}{y_{k}} v_{k} \frac{d q_{k}}{q_{k}}
$$

et (A.2) à :

$$
\frac{d \hat{q}}{\hat{q}}=\sum_{k} v_{k} e_{k} \cdot \frac{d q_{k}}{q_{k}}
$$


où $v_{k}$ est défini par (10) et $e_{k}$ par

$$
e_{k}=\left[1-\frac{\chi_{k}}{y_{k}}\right] \frac{r}{r_{e}}
$$

Introduisant (A.6) et (A.7) dans (A.5) et multipliant par $\left[1-\chi_{h} / y_{h}\right]$ nous obtenons :

$$
\frac{d y_{h}}{y_{h}}=e_{h} \frac{d r}{r}-\sum_{k=1}^{n} C_{h k} \frac{d q_{k}}{q_{k}}
$$

où

$$
C_{h k}=\frac{\sigma r_{e}}{r} e_{h} \delta_{h k}+e_{h} v_{k}\left[1-\frac{\sigma r_{e}}{r} e_{k}\right]
$$

$\delta_{h k}$ étant l'indicateur de Kronecker égal à 1 si $h=k$, à 0 autrement.

L'équation (A.9) montre que $e_{h}$ est l'élasticité-revenu et $-C_{h k}$ une élasticité-prix. Notons encore que, à partir d'ici, nous allons retenir comme variables pertinentes les $e_{h}$ et n'aurons plus à faire intervenir les $\chi_{h}$. De plus, nous notons en passant que les définitions de $v_{h}$ par (10), $r_{e}$ par (A.3) et $e_{h}$ par (A.8) impliquent

$$
\bar{e}=\sum_{h} v_{h} e_{h}=1
$$

En vue d'appliquer le système général (44) à la spécification de Stone-Geary, notons l'expression suivante de l'élément $(j, i)$ de la seconde matrice du membre de droite :

$$
\left\{W D_{v}\left[C{ }^{t} W-e{ }^{t} \bar{w}\right]\right\}_{j i}=\frac{\sigma r_{e}}{r}\left[m\left(e w_{j} w_{i}\right)-m\left(e w_{j}\right) m\left(e w_{i}\right)\right]
$$

où $m\left(e w_{j} w_{i}\right)$ et $m\left(e w_{j}\right)$ sont les moments définis par :

$$
m\left(e w_{j} w_{i}\right)=\sum_{h} v_{h} e_{h} w_{j}^{h} w_{i}^{h} \quad m\left(e w_{j}\right)=\sum_{h} v_{h} e_{h} w_{j}^{h}
$$

Le long d'une isoquante où, par définition ${ }^{t} \bar{w} X^{S}=0$, le système se ramène à :

$$
\frac{r}{r_{e}}\left[D_{\bar{w}} X^{S}\right]_{j}=\sum_{i=1}^{m} J_{j i} \frac{d p_{i}}{p_{i}}
$$

où

$$
J_{j i}=\frac{r}{r_{e}} A_{j i}^{S}-\sigma\left[m\left(e w_{j} w_{i}\right)-m\left(e w_{j}\right) m\left(e w_{i}\right)\right]
$$


Ce système est assez simple puisque nous n'identifions que deux facteurs $(m=2)$, ce qui donne notamment à la matrice $A^{S}$ la forme découlant des équations (64) à (66) du texte. De plus la normalisation des prix des facteurs donnée par (33) peut être prise en compte. Ainsi (A.14) implique :

$$
\frac{r}{r_{e}} \bar{w}_{1} \bar{w}_{2}\left[\frac{d x_{1}^{S}}{x_{1}^{S}}-\frac{d x_{2}^{S}}{x_{2}^{S}}\right]=\left[\bar{w}_{1} \bar{w}_{2}\left(J_{21}+J_{12}\right)-\bar{w}_{2}^{2} J_{11}-\bar{w}_{1}^{2} J_{22}\right]\left[\frac{d p_{2}}{p_{2}}-\frac{d p_{1}}{p_{1}}\right]
$$

La formule permet de lire directement l'élasticité de substitution entre les deux facteurs. Mais elle est trop peu transparente pour nos besoins. En vue d'en dégager les enseignements, considérons d'abord son application à l'économie CES, cas particulier du modèle ici considéré pour le cas où les $e_{h}$ sont tous égaux à 1 et $r_{e}$ égal à $r$. Dans ce cas $m\left(e w_{i}\right)=\bar{w}_{i}$ et le moment $m\left(e w_{j} w_{i}\right)$ est égal à $\operatorname{Cov}\left(w_{i}, w_{j}\right)+\bar{w}_{i} \bar{w}_{j}$. Alors

$$
\begin{aligned}
& J_{11}=J_{22}=-a^{s}-\sigma \operatorname{Var}(w) \\
& J_{12}=J_{21}=a^{s}+\sigma \operatorname{Var}(w)
\end{aligned}
$$

Nous retrouvons exactement l'équation (77) du texte.

Afin de voir en quoi l'hétérogénéité des demandes de biens ajoute ses effets à celle des besoins relatifs en facteurs des diverses branches productives, nous avons naturellement l'idée de décomposer les moments en écrivant par exemple :

$$
\begin{aligned}
& m\left(e w_{1}\right)=m\left[(e-1) w_{1}\right]+\bar{w}_{1} \\
& m\left(e w_{1}^{2}\right)=m\left[(e-1) w_{1}^{2}\right]+\operatorname{Var}(w)+\left(\bar{w}_{1}\right)^{2}
\end{aligned}
$$

Nous pouvons même introduire les moments centrés tels que :

$$
\begin{aligned}
& \bar{m}\left(e w_{1}\right)=\sum_{h} v_{h}\left(e_{h}-1\right)\left(w_{1}^{h}-\bar{w}_{1}\right) \\
& \bar{m}\left(e w_{1}^{2}\right)=\sum_{h} v_{h}\left(e_{h}-1\right)\left(w_{1}^{h}-\bar{w}_{1}\right)^{2}
\end{aligned}
$$

formules dans lesquelles (A.11) est pris en compte. De ces définitions résultent par exemple :

$$
\begin{aligned}
& m\left(e w_{1}\right)=\bar{m}\left(e w_{1}\right)+\bar{w}_{1} \\
& m\left(e w_{1}^{2}\right)=\bar{m}\left(e w_{1}^{2}\right)+2 \bar{w}_{1} \operatorname{Cov}(e, w)+\operatorname{Var}(w)+\left(\bar{w}_{1}\right)^{2}
\end{aligned}
$$

où par définition $\operatorname{Cov}(e, w)$ est identique à $\bar{m}\left(e w_{1}\right)$ défini par (A.20). De (A.22) et (A.23) on déduit directement :

$$
m\left(e w_{1}^{2}\right)-\left[m\left(e w_{1}\right)\right]^{2}=\bar{m}\left(e w_{1}^{2}\right)-\left[\bar{m}\left(e w_{1}\right)\right]^{2}+\operatorname{Var}(w)
$$


Remontant à (A.15) et tenant compte de (31) et (64) à (66), nous obtenons :

$$
J_{11}=-\frac{r}{r_{e}} a^{S}-\sigma \bar{m}\left(e w_{1}^{2}\right)+\sigma[\operatorname{Cov}(e, w)]^{2}-\sigma \operatorname{Var}(w)
$$

Les mêmes calculs peuvent être conduits pour $J_{22}$ et $J_{12}=J_{21}$. Tenant compte de ce que $w_{2}^{h}-\bar{w}_{2}=-\left(w_{1}^{h}-\bar{w}_{1}\right)$, il résulte $\bar{m}\left(e w_{2}^{2}\right)=\bar{m}\left(e w_{1}^{2}\right)=-\bar{m}\left(e w_{1} w_{2}\right)$ et $\bar{m}\left(e w_{2}\right)=-\bar{m}\left(e w_{1}\right)$. Nous trouvons alors :

$$
J_{22}=J_{11} \quad J_{12}=J_{21}=-J_{11}
$$

Ainsi (A.16) se ramène à :

$$
\frac{r}{r_{e}} \bar{w}_{1} \bar{w}_{2}\left[\frac{d x_{1}^{S}}{x_{1}^{S}}-\frac{d x_{2}^{S}}{x_{2}^{S}}\right]=-J_{11}\left[\frac{d p_{2}}{p_{2}}-\frac{d p_{1}}{p_{1}}\right]
$$

Conjointement avec l'expression (A.25) l'élasticité de substitution globale $\sigma^{S}$ s'en déduit :

$$
\sigma^{S}=-\frac{r_{e} J_{11}}{r \bar{w}_{1} \bar{w}_{2}}
$$

Pour faciliter l'interprétation supposons que le moment centré du troisième ordre $\bar{m}\left(e w_{1}^{2}\right)$ soit nul et que les $\sigma^{h}$ soient tous égaux au même $\sigma^{P}$. Alors

$$
-J_{11}=\frac{r}{r_{e}} \cdot \sigma^{P}\left[\bar{w}_{1} \bar{w}_{2}-\operatorname{Var}(w)\right]+\sigma^{C} \operatorname{Var}(w)-\sigma^{C}[\operatorname{Cov}(e, w)]^{2}
$$

D'où :

$$
\sigma^{S}=\sigma^{P}+\left[\frac{r_{e}}{r} \sigma^{C}-\sigma^{P}\right] \frac{\operatorname{Var}(w)}{\bar{w}_{1} \bar{w}_{2}}-\frac{r_{e} \sigma^{C}}{r \bar{w}_{1} \bar{w}_{2}}[\operatorname{Cov}(e, w)]^{2}
$$

Le membre de droite est inférieur à celui donné par (79) en raison de la soustraction du dernier terme, lequel est normalement positif, et du fait que, dans le second terme, $\sigma^{C}$ est multiplié par la fraction $r_{e} / r$ normalement plus petite que 1 .

Ainsi que nous l'avons fait à la fin de la section 7, considérons maintenant le cas où, le second facteur étant le travail non-qualifié, il existerait une offre excédentaire de ce travail conjointement avec un prix $p_{2}$ exogène. Les variables exogènes seraient alors $x_{1}^{S}$ et $p_{2}$ tandis que la demande globale de travail non-qualifié, maintenant écrite $x_{2}^{D}$, serait endogène. Notre objectif consiste à dégager la formule qui lie $d x_{2}^{D} / x_{2}^{D}$ à $d p_{2} / p_{2}$ quand $x_{1}^{S}$ ne varie pas. Pour cela nous devons appliquer la formule générale (44) en tenant compte de ce que la normalisation des prix implique, selon (33) : 


$$
\frac{d p_{1}}{p_{1}}=-\frac{\bar{w}_{2}}{\bar{w}_{1}} \cdot \frac{d p_{2}}{p_{2}}
$$

De même que (A.12) va nous servir à appliquer l'expression du membre de droite de (44), nous pouvons appliquer l'expression du membre de gauche en observant que :

$$
\left\lfloor W D_{v} e^{t} \bar{w}\right\rfloor_{j i}=m\left(e w_{j}\right) \bar{w}_{i}
$$

comme la première composante de $X^{S}$ est nulle ( $x_{1}^{S}$ ne varie pas), la première ligne de (44) s'écrit :

$$
-m\left(e w_{1}\right) \bar{w}_{2} \frac{d x_{2}^{D}}{x_{2}^{D}}=\frac{r_{e}}{r}\left[J_{11} \frac{d p_{1}}{p_{1}}+J_{12} \frac{d p_{2}}{p_{2}}\right]
$$

ou encore, compte tenu de (A.26) et (A.31) :

$$
-m\left(e w_{1}\right) \bar{w}_{1} \bar{w}_{2} \frac{d x_{2}^{D}}{x_{2}^{D}}=\frac{-r_{e}}{r} J_{11} \frac{d p_{2}}{p_{2}}
$$

Les formules (A.22) et (A.28) conduisent alors à :

$$
\frac{d x_{2}^{D}}{x_{2}^{D}}=-\frac{\sigma^{S}}{\bar{w}_{1}+\operatorname{Cov}(e, w)} \cdot \frac{d p_{2}}{p_{2}}
$$

Le multiplicateur de $d p_{2} / p_{2}$ est d'autant plus élevé en valeur absolue que $\sigma^{S}$ est plus élevé et que $\operatorname{Cov}(e, w)$ est plus négative. 


\section{Références}

D. Acemoglu (2002), «Technical change, inequality and the labor market », Journal of Economic Literature, March, p. 7-72.

A. Bernard (1986), "Mesure et représentation de la substituabilité", Communication au Séminaire René Roy, Paris.

W.E. Diewert and A.D. Woodland (1977), «Frank Knight's theorem in linear programming revisited », Econometrica, March 1977, Vol 45, p. 375-98.

D.R. Helm (1987), "Elasticity of substitution", dans The New Palgrave Dictionary of Economics, Macmillan, London.

E. Malinvaud (2001), «An aggregation problem : the demand for unskilled labour », Document de travail CREST, $\mathrm{n}^{\circ}$ 2001-22.

E. Malinvaud (2002), "Sur l'agrégation des demandes de travail non-qualifié", Annales d'Economie et de Statistique, N 66, p. 42-80.

R. Stone (1954), «Linear expenditure systems and demand analysis : an application to the pattern of British demand », Economic Journal, vol. 64, p. 511-27. 